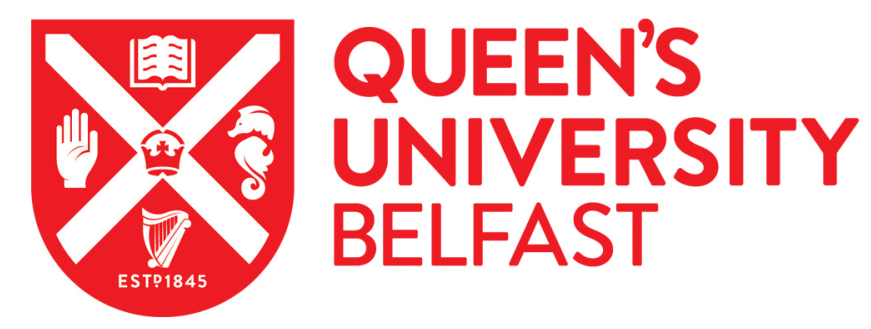

\title{
Effectiveness of prosthodontic interventions and survival of remaining teeth in adult patients with shortened dental arches - a systematic review
}

McLister, C., Donnelly, M., Cardwell, C. R., Moore, C., O'Neill, C., Brocklehurst, P., \& McKenna, G. (2018). Effectiveness of prosthodontic interventions and survival of remaining teeth in adult patients with shortened dental arches - a systematic review. Journal of Dentistry. https://doi.org/10.1016/j.jdent.2018.02.003

Published in:

Journal of Dentistry

Document Version:

Peer reviewed version

Queen's University Belfast - Research Portal:

Link to publication record in Queen's University Belfast Research Portal

\author{
Publisher rights \\ Copyright 2018 Elsevier. \\ This manuscript is distributed under a Creative Commons Attribution-NonCommercial-NoDerivs License \\ (https://creativecommons.org/licenses/by-nc-nd/4.0/), which permits distribution and reproduction for non-commercial purposes, provided the \\ author and source are cited.
}

\section{General rights}

Copyright for the publications made accessible via the Queen's University Belfast Research Portal is retained by the author(s) and / or other copyright owners and it is a condition of accessing these publications that users recognise and abide by the legal requirements associated with these rights.

Take down policy

The Research Portal is Queen's institutional repository that provides access to Queen's research output. Every effort has been made to ensure that content in the Research Portal does not infringe any person's rights, or applicable UK laws. If you discover content in the Research Portal that you believe breaches copyright or violates any law, please contact openaccess@qub.ac.uk. 


\section{Title page}

2 Full Title: Effectiveness of prosthodontic interventions and survival of remaining teeth

3 in adult patients with shortened dental arches - a systematic review

4 Short title: Prosthodontic interventions in shortened dental arches

5 Authors: Conor McLister ${ }^{1}$, Michael Donnelly ${ }^{1}$, Christopher R Cardwell ${ }^{1}$, Ciaran Moore ${ }^{1}$,

6 Ciaran 0’Neill ${ }^{1}$, Paul Brocklehurst ${ }^{2}$, Gerald McKenna ${ }^{1}$

7 Affiliations: ${ }^{1}$ Centre for Public Health, Queen's University Belfast, United Kingdom,

$8 \quad$ 2NWORTH Clinical Trials Unit, North Wales

9 Word count: 4022

10 N figures: 4

11 N tables: 5

12 Keywords: systematic review, shortened dental arch, removable partial dental

13 prostheses, randomized clinical trial

14 Corresponding author:

15 Gerald McKenna

16 Centre for Public Health

17 Queen's University Belfast

18 Institute of Clinical Sciences Block B

19 Grosvenor Road

20 Belfast

21 BT12 6BJ

22 United Kingdom

23 g.mckenna@qub.ac.uk

24 Tel: +442890978999

25 Fax: +442890235900 


\section{Abstract (242/ 250 words)}

2 Objectives: A systematic review of randomised and non-randomised controlled trials

3 was conducted to evaluate studies of the effectiveness of different tooth replacement

4 strategies in adult patients with shortened dental arches. The objectives of the review

5 were to determine the survival rates of different prosthodontic interventions, the risk of

6 tooth loss with and without prosthodontic interventions, and the impact of different

7 tooth replacement strategies on oral-health related quality of life (OHRQoL).

8 Methods: The protocol was registered with the International Prospective Register of

9 Systematic Reviews (PROSPERO CRD42017064851), and the review was conducted in

10 accordance with the guidelines of the Preferred Reporting Items for Systematic Reviews

11 and Meta-analyses (PRISMA).

12 Results: The search strategy identified 112 potentially relevant publications; 22 from

13 Medline (OVID), 54 from EMBASE (OVID), 35 from CENTRAL, one from the authors'

14 knowledge of the subject area, and none from OpenSIGLE. Ten articles were included in

15 this systematic review. Of these, four were analyses of different outcomes from a

16 multicentre randomized controlled trial in Germany, whilst one study was the pilot

17 phase for this trial. Two further randomized controlled trials were included from the

18 United Kingdom and Republic of Ireland. The remaining articles were reports of

19 prospective cohort studies from Denmark and the Netherlands.

20 Conclusions: there is currently insufficient evidence to recommend one tooth

21 replacement strategy over another in adult patients with reduced dentitions.

22 Clinical significance: (44/ 50 words) There is a need for further research as there are

23 insufficient numbers of good quality randomised controlled trials currently available.

24 Authors should be encouraged to adhere to CONSORT guidelines for randomized

25 controlled trials, and report findings in such a way that facilitates future meta-analysis. 


\section{Introduction}

2 The population of the world is ageing. The United Nations has estimated that globally,

3 the percentage of older persons (60 years and over) increased from $9.9 \%$ in 2000 to

$4 \quad 12.3 \%$ in 2015 . It is expected that this percentage will rise to over $20 \%$ by 2050 , with an

5 elderly population of nearly 2.1 billion (Fig. 1). ${ }^{1}$ As significant transformations are

6 occurring in populations, changes have also been noted in oral health. More and more

7 adults are retaining their natural teeth into old age (Fig. 2). The 2009 UK Adult Dental

8 Health Survey (ADHS) reported that only 6\% of those surveyed were missing all their

9 teeth, a significant decrease from $37 \%$ in $1968 .^{2}$

11 With increased tooth retention, population growth and ageing, the global burden of oral

12 conditions has increased by approximately $20.8 \%$ since 1990 . Collectively, oral

13 conditions affected 3.9 billion people worldwide in 2010, with untreated caries and

14 severe periodontal disease causing an increased burden, especially in less developed

15 regions. These oral conditions often lead to becoming partially dentate. 4

16

17

Potential consequences of tooth loss include impaired mastication, altered food choices, psychosocial problems and reduced oral health related quality of life.5,6 However, depending on the pattern of tooth loss, it may not be necessary to replace all missing teeth, especially in older patients. Kayser first described the shortened dental arch (SDA) concept, suggesting that patients with at least four occlusal units (one unit = pair of occluding premolars; two units = pair of occluding molars) had sufficient adaptive capacity to constitute a functional dentition. ${ }^{7}$ The concept has been suggested as an oral health goal for adults until the end of life by the World Health Organisation, ${ }^{8}$ and is considered to have a useful role in contemporary clinical practice. ${ }^{9}$

Where tooth replacement is required to restore partially dentate patients to at least a 
1 reduced functional dentition, there are various fixed and removable prosthetic options.

2 Traditionally these have included removable partial dentures, and resin bonded or

3 conventional bridgework. In the last number of decades these options have grown in

4 scope with the demonstrated predictability of dental implants. However, decision

5 making for different patterns of tooth loss and patient groups is often not evidence

6 based. ${ }^{10}$ In addition, the financial cost of tooth loss disproportionately affects older age

7 groups ${ }^{11}$, and there is a need to achieve better clinical outcomes, which are cost-effective

8 and require less maintenance.

9

A recent systematic review concluded that the shortened dental arch concept appears to

$\underline{\text { be as feasible as tooth replacement with removable partial dentures in partially dentate }}$ patients. ${ }^{12}$ However, outcome measures were restricted to the impact on oral health related quality of life. Thus, a more comprehensive systematic review of randomised and non-randomised controlled trials was conducted to evaluate studies of the oral-health related quality of life (OHRQoL).

\section{Material and Methods}

Methods of analysis and inclusion criteria for this systematic review were specified in advance and published as a protocol. ${ }^{13}$ The protocol was registered with the International Prospective Register of Systematic Reviews (PROSPERO

25 CRD42017064851), and the review was conducted in accordance with the guidelines of 26 the Preferred Reporting Items for Systematic Reviews and Meta-analyses (PRISMA) ${ }^{14}$. 
2 Eligibility criteria included experimental or observational study designs investigating

3 partially dentate adult (18 years or older) patients with between 4 and 10 functional

4 teeth in occlusion with a natural dentition or prosthesis. Functional teeth in the maxilla,

5 mandible or both arches were eligible for inclusion. Eligible prosthodontic

6 interventions were removable partial dentures, conventional or resin bonded

7 bridgework, implant supported crown or bridgework, and the comparator was no

8 intervention or different interventions ('head-to-head'). Primary outcomes included

9 survival of prosthodontic interventions (mean follow-up of 5 years or more), survival of

10 remaining teeth (mean follow-up of 5 years or more) and change in OHRQoL using

11 validated self-reported measures (mean follow-up of 1 year or more). Secondary

12 outcomes included any biological or technical complications.

14 The electronic databases of MEDLINE, CENTRAL, Embase and the grey literature

15 database of OpenSIGLE were searched for primary studies conducted in the period from

161980 to and including $1^{\text {st }}$ November 2017. The OVID interface (MEDLINE, Embase)

17 search strategy is available in Appendix 1, and this was adapted for CENTRAL as

18 appropriate. The trial registries of the World Health Organisation (ICTRP) and US

19 National Institutes of Health (ClinicalTrials.gov) were also searched. Reference lists of

20 included studies or reviews identified through the search were reviewed for any further

21 eligible studies. All searches were restricted to articles published in the English

22 language.

23

24 Two review authors (CML and CM) extracted data from each included study

25 independently and in duplicate using a data collection sheet developed for the review.

26 Any differences were resolved by discussion and, where necessary, arbitration by a 
1 third person (GMK). For each study the following data was recorded: year of

2 publication, country of origin, funding; participants; study design; outcomes.

3

\section{Results}

\section{Study selection}

6 Two independent review authors (CML and CM) screened all titles and abstracts

$7 \quad$ identified by the electronic searches. Full reports were obtained for all titles that

8 appeared to meet the inclusion criteria or where there was uncertainty. Disagreements

9 between reviewers were resolved by discussion, and a third reviewer (GMK) was

10 available for resolution of any differences. As described in the PRISMA flow diagram

11 (Fig. 3), the search strategy identified 112 potentially relevant publications; 22 from

12 Medline (OVID), 54 from EMBASE (OVID), 35 from CENTRAL, one from the authors'

13 knowledge of the subject area, and none from OpenSIGLE. After 32 duplicates were

14 identified, 80 titles and abstracts were screened by both reviewers independently.

15 Inter-rater reliability was assessed using the Kappa statistic, with substantial agreement

16 between the reviewers $-\mathrm{K}=0.68$ (95\% CI 0.51, 0.85). Following discussion, and

17 arbitration by the third reviewer, 60 of these citations were excluded. Subsequently,

18 twenty full text articles were retrieved and screened. From this, ten studies were

19 eligible for inclusion in this systematic review. The main characteristics of each

20 included study are presented in Table 1 . Full reports that were excluded are presented

21 in Table 2.

23 An initial evaluation of the included papers showed considerable heterogeneity in study

24 populations, interventions and outcome measures. Despite clinical heterogeneity a meta-analysis was undertaken for the outcome 'survival of prosthodontic interventions'. 
1 This was not considered appropriate for other outcomes, and therefore a descriptive manner of data presentation was used.

\section{Study populations}

Budtz-Jorgensen and Isidor ${ }^{15}$ followed 53 patients at the Royal Dental College, Aarhaus, Denmark, who had complete maxillary dentures opposed by partially dentate mandibles. Twenty-five of these were male and twenty-eight were female. Mean ages in the study groups were 69.7 years (range 61 - 83) and 68.3 years (range 61 - 81), whilst the mean number of mandibular teeth in each group was 6.9 (SD 1.7) and 7.5 (1.7). In the Netherlands, Gerritsen et al.18,19, analysed the records of 59 patients participating in a prospective observational cohort study at the Nijmegen Dental School. Of these patients, twenty-one were male and thirty-eight were female. The study cohort comprised patients with shortened dental arches in at least one jaw (intact anterior dentitions and 3-4 posterior occluding pairs), shortened dental arches extended by removable partial dentures and a control group with complete dental arches. The average ages at baseline in the respective groups were 37.8 years (SD 11.2), 31.7 years (SD 8.0) and 40.0 years (SD 9.7).

Thomason et al. ${ }^{17}$ recruited 60 patients at Newcastle Dental Hospital, United Kingdom, who had a maximum of eight remaining mandibular teeth, excluding molars. Twentyfive of these patients were male and thirty-five female, with a median age of 67 years (range $39-81$ ). In a pilot study, Wolfart et al. ${ }^{16}$ recruited 30 patients at a German dental school who were also missing molars in one jaw, and at least one canine and one premolar present bilaterally. There was equal recruitment of males and females, with a mean age of 62 years. In the subsequent multi-centre randomized controlled trial, 
1 in Germany. Inclusion criteria for remaining teeth was as for the pilot phase of the

2 study. ${ }^{16}$ Allocated study groups had mean ages of 60.4 years (SD 10.6) and 59.6 years

3 (SD 10.4), with 70 males and 82 females participating. Most recently McKenna et al.24

4 recruited 132 patients from a university dental hospital and a geriatric day hospital in

5 the Republic of Ireland. Recruitment was restricted to patients over 65 years seeking

6 tooth replacement, who had a minimum of 6 remaining natural teeth in both arches of

7 good prognosis. Neither the specific age profile or gender of the participants was

8 reported.

9

All of the included studies investigated removable partial dentures as an intervention in a study arm. ${ }^{15-24}$ Conventional cobalt chrome metal frameworks were provided for patients in three of the studies ${ }^{15,17,24}$, whilst removable partial dentures in the pilot phase and subsequent multicentre randomized controlled trial in Germany were retained by precision attachments. 16,20-23 Specific design features of removable partial dentures were not reported by Gerritsen et al. ${ }^{18,19}$ All of the studies also investigated fixed tooth replacement to at least a shortened dental arch, if not already present. Cantilever fixed partial dentures were used to restore patients in one arm of the German study. ${ }^{16,20-23}$ Budtz-Jorgensen investigated fixed partial dentures retained by pins and boxes, with single and double abutment and pontic designs up to ten units. ${ }^{14}$ In the studies by McKenna et al. and Thomason et al.17,24, more minimally invasive resin bonded bridges were investigated, whilst Gerritsen et al. ${ }^{18,19}$ included a third control group of patients with complete dental arches for comparison. Intervention with implant supported crown or bridgework was not analyzed by any of the included studies. 
3 Two studies assessed survival of prosthodontic interventions after 5 years. Budtz-

$4 \quad$ Jorgensen and Isidor ${ }^{15}$ reported number of prosthesis failures whilst Thomason et al. ${ }^{17}$

5 reported survival probability and compared interventions using hazard ratios. Survival

6 of remaining teeth was analyzed in four studies but outcome measures varied. Budtz-

7 Jorgensen and Isidor ${ }^{15}$ reported the number of tooth extractions in each study group

8 over a 5-year follow-up period. Gerritsen et al. reported cumulative survival and hazard

9 ratios for tooth loss with a mean follow up of $27.4-35$ years ${ }^{18}$, whilst a separate

10 analysis reported the rate of tooth loss ${ }^{19}$. Walter et al. reported survival probability for

11 tooth loss in both jaws, the study jaw and in relation to most posterior teeth at 5 years ${ }^{20}$.

12 Three studies provided data on changes in oral health related quality of life (OHRQoL).

13 Wolfart et al. measured changes using the OHIP-49 questionnaire in a pilot study over

1412 months $^{16}$, and subsequently used the same measure in a multi-centre trial with 5 -

15 year follow-up ${ }^{21}$. More recently, Mc Kenna at al. used OHIP-14 questionnaires to assess

16 the impact of treatments over a 12-month period 24 . Several studies reported different

17 secondary outcomes over a minimum follow-up period of 5 years. Outcome measures

18 included cumulative survival and hazard ratios for first restorative interventions, rate of

19 restorative interventions, changes in periodontal indices, incidence of caries and

20 number of treatments for biological and technical reasons. $15,18,19,22,23$

Quality assessment

24 Cochrane risk of bias ${ }^{35}$ assessments were undertaken of each randomized controlled

25 trial report included. These are presented in Table 3, and a summary of the overall

26 quality of these studies is shown in Fig. 4. The quality of three included non-

27 randomized, non-interventional studies was assessed using the Newcastle Ottawa 
1 Scale ${ }^{36}$ protocol. Of these, the study by Budtz-Jorgensen ${ }^{15}$ was assessed as being of the

2 best quality, earning 8 out of 9 stars for cohort studies. The cohort studies by Gerritsen

3 et al. earned $6^{19}$ and $7^{18}$ stars respectively, across the domains of selection,

4 comparability and outcome.

5

\section{Conclusions of included studies}

\section{Survival of prosthodontic interventions}

Thomason et. al ${ }^{17}$ reported survival probabilities of approximately $25 \%$ for removable partial dentures and 70\% for resin bonded bridges at 5 years. Resin bonded bridges had a slightly lower hazard rate, but the difference was not statistically significant (Hazard ratio $=0.59 ; 95 \%$ CI $0.27,1.29)$. Significantly, patients in the resin bonded bridge group also required less treatment intervention at follow-up appointments (39/175) compared with the removable partial denture group (78/175). Accepting a loss of power in the study, the authors concluded that the greater need for maintenance in the RPD group, the reported advantages of resin bonded bridges ${ }^{29,37}$ and the absence of significant difference in survival, offers positive support for the use of resin bonded bridges in restoring shortened lower dental arches of elderly persons. Previously, Budtz-Jorgensen at el. ${ }^{15}$ also concluded that treatment with distally extending cantilevered fixed partial dentures is a favourable alternative to treatment with RPDs in elderly patients. There were relatively more failures in the removable partial denture group $(10 / 26)$ than in the fixed partial denture group (8/41) over the 5 year period, but no statistical analysis was undertaken.

\section{Survival of remaining teeth}


1 In their prospective cohort study, Budtz-Jorgensen and Isidor ${ }^{15}$ reported more

2 extractions in the RPD study group (11) than in the fixed partial denture group (1)

3 during 5 year follow-up. However, as with prostheses survival, no statistical analysis

4 was undertaken. When comparing shortened dental arches with and without

5 removable partial dentures, Gerritsen et al. ${ }^{19}$ found no significant difference in

6 cumulative survival of remaining anterior or premolar teeth. However, the authors

7 concluded that patients with a shortened dental arch had an increased risk of losing

8 premolar teeth, as the hazard ratio was statistically significant when compared to the

9 complete dental arch group. In a further analysis, Gerritsen et al. ${ }^{18}$ reported no

10 statistically significant difference in the per year risk of tooth loss between the

11 shortened dental arch groups with or without removable partial dentures. However,

12 they concluded that replacement of absent posterior teeth by free end removable partial

13 dentures cannot be recommended as it seems to be associated with a less favourable

14 clinical course. Walter et al. ${ }^{20}$ also found no significant differences in survival

15 probability at 5 years for first tooth loss in both jaws, the study jaw or in relation to

16 most posterior teeth, with or without removable partial dentures.

17

\section{Changes in Oral Health Related Quality of Life}

In Germany, Wolfart et al. ${ }^{16,21}$ compared the impact on OHRQoL for patients with and without removable partial dentures. Both a pilot study ${ }^{16}$ and subsequent multicentre randomized controlled trial ${ }^{21}$, concluded that both treatment concepts showed a similar improvement in OHRQoL, with no significant differences between the treatment groups. The multicentre study did note a slightly longer adaptation period in the removable partial denture group, with improvements in OHRQoL continuing until 1 year postinsertion. In contrast, McKenna et al. ${ }^{24}$ concluded that treatment based on the SDA concept achieved significantly better results than that based on RPDs, in terms of impact 
1 on OHRQoL. These results were seen in both a dental hospital and geriatric day hospital

2 setting, 12 months after treatment intervention.

3

\section{Biological and Technical Complications}

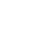

Budtz-Jorgensen and Isidor ${ }^{15}$, when comparing FPDs to RPDs, concluded that generally the need for dental and prosthetic follow-up treatment was more pronounced in the RPD group than in the FPD group. Fifty-seven carious lesions were observed in the RPD group compared with 10 lesions in the FPD group, although again statistical analysis was not undertaken. They also noted no progression of periodontal disease adjacent to the abutment teeth in any of the groups. Walter et al.23 did find statistically significant although minor detrimental effects of RPDs on periodontal health, when compared to patients restored to a fixed premolar occlusion. Overall, small significant differences were noted in plaque indices, bleeding indices, clinical attachment loss and probing pocket depths in distal sites of the posterior most teeth associated with prostheses.

$$
\text { However, the authors concluded that these small negative effects do not justify a }
$$
rejection of RPDs when they are indicated. From the same German study, Wolfart et al. ${ }^{22}$ found statistically significant differences in treatment for technical reasons over the 5year follow-up. $24 \%$ of patients in the RDP group needed treatment compared with $8 \%$ in the SDA group ( $\mathrm{p}=0.01)$. In the analysis by Gerritsen et al. ${ }^{19}$, the authors concluded that wearing a RPD in SDA subjects did not increase the risk of receiving a first-time restoration. However, SDA subjects did have an increased risk of receiving a first-time restoration in anterior and premolar teeth compared to complete dental arch subjects. In a separate analysis ${ }^{18}$, they also found no statistically significant difference in the per year risk of direct, indirect restorations or endodontic treatments, between the shortened dental arch groups with or without removable partial dentures. 


\section{Discussion}

2

3 Ten articles were included in this systematic review. Of these, four were analyses of

4 different outcomes from a multicentre randomized controlled trial in Germany, whilst

5 one study was the pilot phase for this trial. Two further randomized controlled trials

6 were included from the United Kingdom and Republic of Ireland. The remaining articles

7 were reports of prospective cohort studies from Denmark and the Netherlands.

8

9 Only two studies considered the survival of prosthodontic interventions in adult patients with shortened dental arches after a minimum follow-up period of 5 years. This time period was chosen as it has been used in other systematic reviews investigating indirect prostheses. ${ }^{38-40}$ However, it is accepted some clinicians may argue that such a period is too short to obtain reliable information on survival and complication rates. ${ }^{41}$ Both studies compared cantilever bridgework to removable partial dentures. Meta-analysis (Figure 5) showed statistically significant better survival for cantilever bridgework. However this should be interpreted with caution, due to the noted clinical heterogeneity between these studies. All patients in the study by Budtz-Jorgensen and Isidor had maxillary complete dentures and more invasive bridge designs were used in the mandible. Restorations were also cemented with a luting cement (Zinc Phosphate) and therefore, the data may not reflect the performance of more contemporary resin bonded materials. Thomason et al. did use more contemporary resin bonding techniques and single abutments wherever possible. Such techniques for cantilever resin bonded bridges are associated with relatively high survival rates ${ }^{42}$, in comparison with removable partial dentures at 5 and 10 years. ${ }^{43}$ This study failed to detect a statistically significant difference in time to survival between the two treatment groups, although the RPD group required significantly more treatment interventions and maintenance at follow-up appointments. Again these 
1 findings should be interpreted with caution, as the small sample size and relatively high

2 drop-out (15 patients) is likely to have resulted in loss of power and ability to show any

3 true difference between the interventions. The German multicentre study also reported

4 more maintenance for technical reasons in the RPD group, although they were retained

5 by precision attachments, which would not be standard practice in the United Kingdom.

6 In addition, they reported significant but minor detrimental effects of RPDs on

7 periodontal health. Previous studies have shown increased plaque and gingivitis,

8 particularly at abutment teeth, and these results may reflect the less hygienic, more

9 complex design used. However, there is no clear evidence that RPDs increase the risk of periodontitis. ${ }^{44}$

Tooth loss was considered in four of the included studies. Budtz-Jorgensen and Isidor reported more extractions in the RPD group than the FPD group. However, it was suggested that several of these teeth could have been retained if patients had been willing to accept more costly further treatment. This highlights how cost, amongst other factors, can be a barrier to treatment and cause inequality in dental service utilisation ${ }^{12,45}$. Gerritsen et al., in separate analyses of a prospective cohort study, reported that for patients with shortened dental arches, wearing removable partial dentures had no significant impact on cumulative survival of remaining teeth or risk of tooth loss. However, when compared to a third group of patients with complete dental arches, cumulative survival of premolar teeth in patients with shortened dental arches was significantly lower. Again, these results must be interpreted with caution due to the small sample size, and no detail of possible confounding variables such as previous caries status, smoking, diet or oral hygiene. The multicentre RCT in Germany also found no significant difference in cumulative survival at 5 years for tooth loss in each study

26 group. In general, these findings are consistent with the understanding of tooth loss as a multifactorial outcome that is difficult to predict. 46,47 
2 It is recognised that purely clinical indicators are insufficient when assessing treatment

3 outcomes. For treatment plans to meet patient preferences and needs, the gap between

4 the clinican's and patient's view of clinical reality must be narrowed. Many subjective

5 patient reported outcome measures (PROMS) have been developed, but few are used

6 routinely at the point of care. ${ }^{48}$ Wolfart et al. and McKenna et al. used different versions

7 of the oral health impact profile (OHIP) to assess changes in oral health related quality

8 of life (OHRQoL) in their randomized controlled trials. This is a widely reported and

9 validated tool ${ }^{49-51}$, with versions including 49 item (OHIP-49) and 14 item (OHIP-14)

10 questionnaires. There is strong evidence that tooth loss is associated with impairment

11 in OHRQoL, however, the prevalence of negative impacts increases significantly when

12 the number of occluding pairs of teeth drops below ten. ${ }^{6}$ McKenna et al. ${ }^{24}$ found that

13 treatment according to the SDA concept resulted in significantly better mean OHIP-14

14 scores compared with RPD treatment, in both a dental hospital and geriatric day

15 hospital setting. Contemporary standardised protocols were used for provision of resin

16 bonded bridges in the SDA group and cobalt chrome frameworks were provided in the

17 RPD group. In contrast, Walter et al.16,21 used median OHIP-49 scores in both studies,

18 and found no significant differences between the SDA and RPD groups at 12 months or

19 at 5 years. These findings were similar to a previous UK pilot study, comparing the SDA

20 concept with RPDs. Summary satisfaction scores improved in both groups, but

21 significant differences were not established. ${ }^{29}$

23 A major limitation of this review is that it was only possible to conduct a meta-analysis

24 using two studies for one outcome, and the overall estimate of treatment effect is

25 therefore limited. This reflects the considerable heterogeneity in interventions and

26 outcomes across only ten included studies. Heterogeneity makes it difficult to compare

27 inconsistency, indirectness and imprecision across studies. In general, the quality of 
1 studies varied. This is consistent with a previous review of restorative approaches in

2 shortened dental arch patients, which graded the overall body of evidence as low. ${ }^{2}$ In

3 our review, randomization was judged to be adequate in all trials. However, for indirect

4 prostheses it is almost impossible to blind the clinician or patient from the intervention,

5 whilst blinding of the assessor is challenging due to marked differences in the

6 appearance of prostheses. All but one of the included randomized trials were assessed

7 as at high risk of performance bias, but lack of blinding was considered unlikely to affect

8 outcomes in the majority of studies. Both the United Kingdom and German multicentre

9 trials experienced significant numbers of patients lost to follow-up, and loss of power,

10 whilst the cohort studies also had small sample sizes. Another limitation is that the

11 review was mainly based on studies that were conducted in an institutional

12 environment, such as university or hospital based clinics, and therefore lacks external

13 validity. It is important to note that not all possible prosthodontic interventions were

14 considered, with no studies on dental implants included. Furthermore, some of the

15 prosthodontic interventions provided, particularly in the Danish and German studies,

16 are much more invasive than would be considered standard practice in the United

17 Kingdom. All searches included only English-language publications, and this may have

18 excluded several additional studies published in other languages. However, the scoping

19 exercise suggested this was unlikely and previous studies ${ }^{53}$ have found little effect in

20 excluding trials published in languages other than English, on combined effect estimates

21 in meta-analyses of RCTs.

\section{Conclusion}

In conclusion there is currently insufficient evidence to recommend one tooth replacement strategy over another in adult patients with reduced dentitions. There is limited evidence that removable partial dentures are associated with more maintenance 
1 and impact less on oral health related quality of life, in comparison with restoration to a

2 shortened dental arch using resin bonded bridges. However, there is a need for further

3 research as there are insufficient numbers of good quality randomised controlled trials

4 currently available. Authors should be encouraged to adhere to CONSORT guidelines for

5 randomized controlled trials, and report findings in such a way that facilitates future

6 meta-analysis. In particular, future studies should focus on contemporary

7 prosthodontic interventions, including dental implants, and provide more standardised

8 core outcomes with longer term follow-up. These should include subjective qualitative

9 outcomes so that future treatment strategies can be based on evidence that is 'patient

10 centred'. Finally, with an aging population, and evidence of income related barriers to

11 oral healthcare for many older adults ${ }^{11}$, there is a need to ascertain which treatment

12 strategies are most cost-effective.

\section{Acknowledgements}

15 Conflicts of interest: none. Funding by the Royal College of Surgeons Edinburgh, Small

16 Research Grant. 


\section{Tables}

\section{Table 1:}

Characteristics of included studies

\begin{tabular}{|c|c|c|c|c|c|c|c|}
\hline & Setting & Participants & Age & Country & Interventions & Outcomes & $\begin{array}{l}\text { Follow- } \\
\text { up }\end{array}$ \\
\hline $\begin{array}{l}\text { Budzt- } \\
\text { Jorgensen et } \\
\text { al. }(1990)^{15}\end{array}$ & DS & 53 & $61-83$ & Denmark & $\begin{array}{l}\text { SDA / FPD } \\
\text { RPD }\end{array}$ & $\begin{array}{l}\text { Intervention } \\
\text { Survival } \\
\text { Biological / } \\
\text { technical } \\
\text { complications }\end{array}$ & 5 years \\
\hline $\begin{array}{l}\text { Wolfart et al. } \\
(2005)^{16}\end{array}$ & DS & 30 & $>35$ & Germany & $\begin{array}{l}\text { SDA / FPD } \\
\text { RPD }\end{array}$ & OHRQoL & 1 year \\
\hline $\begin{array}{l}\text { Thomason et } \\
\text { al. }(2007)^{17}\end{array}$ & $\mathrm{DH}$ & 60 & $39-81$ & UK & $\begin{array}{l}\text { SDA / RBB } \\
\text { RPD }\end{array}$ & $\begin{array}{l}\text { Intervention } \\
\text { Survival }\end{array}$ & 5 years \\
\hline $\begin{array}{l}\text { Gerritsen et } \\
\text { al. }(2013)^{18}\end{array}$ & DS & 59 & $\begin{array}{l}\text { Mean } \\
\text { ages: } \\
37.8 \\
(11.2) \text {, } \\
31.7 \\
(8.0), \\
40.0 \\
(9.7)\end{array}$ & Netherlands & $\begin{array}{l}\text { SDA / FPD } \\
\text { CDA } \\
\text { RPD }\end{array}$ & $\begin{array}{l}\text { Tooth } \\
\text { Survival } \\
\text { Biological / } \\
\text { technical } \\
\text { complications }\end{array}$ & $\begin{array}{l}27-35 \\
\text { years }\end{array}$ \\
\hline $\begin{array}{l}\text { Gerritsen et } \\
\text { al. }(2013)^{19}\end{array}$ & DS & 59 & $\begin{array}{l}\text { Mean } \\
\text { ages: } \\
37.8\end{array}$ & Netherlands & $\begin{array}{l}\text { SDA / FPD } \\
\text { CDA } \\
\text { RPD }\end{array}$ & $\begin{array}{l}\text { Tooth } \\
\text { Survival } \\
\text { Biological / }\end{array}$ & $\begin{array}{l}27-35 \\
\text { years }\end{array}$ \\
\hline
\end{tabular}




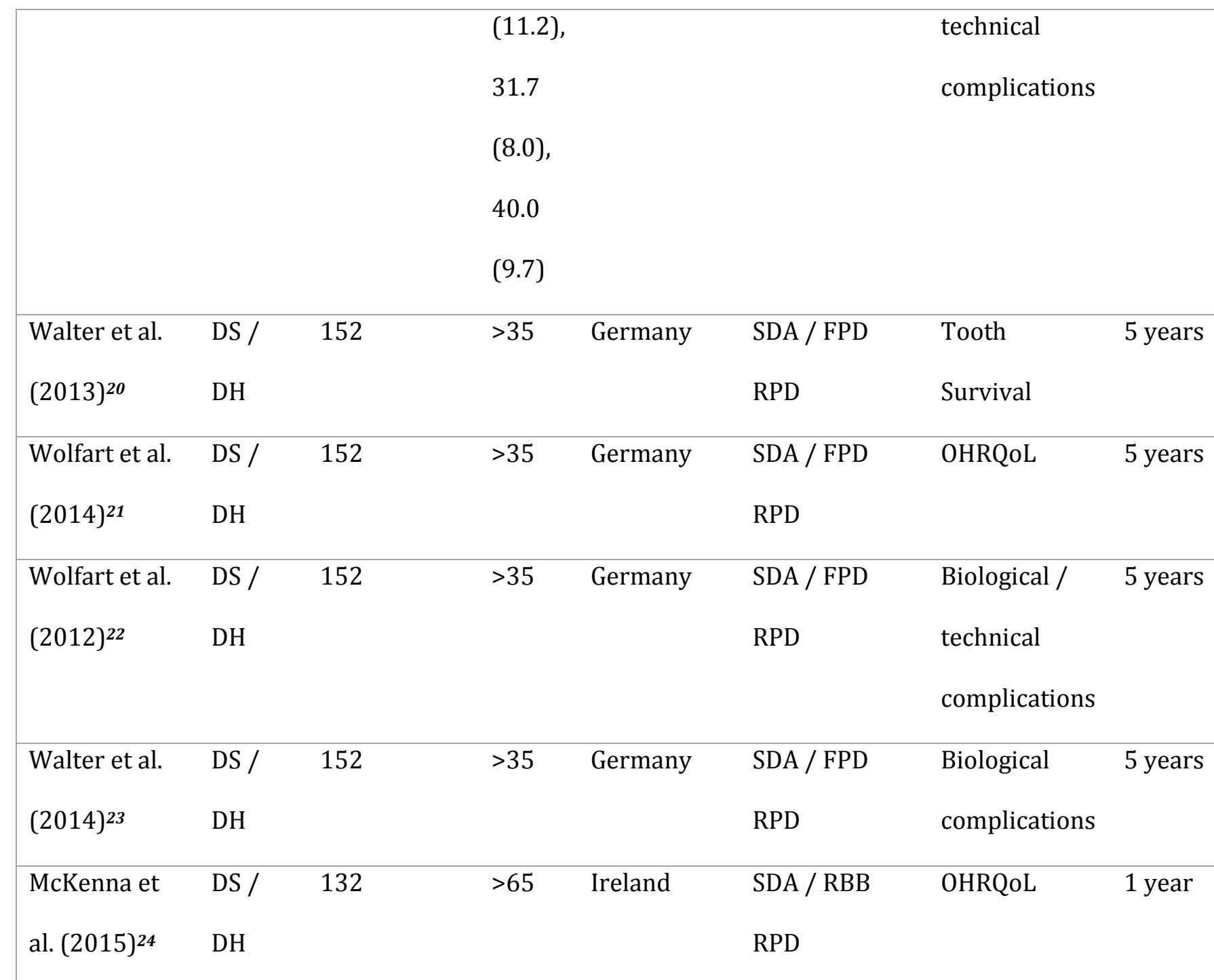

\section{Table 2}

Characteristics of excluded studies

\section{Study Reason for exclusion}

\begin{tabular}{|c|c|}
\hline Baba et al. (2008) ${ }^{25}$ & $\begin{array}{l}\text { Study of cross sectional design with no intervention } \\
\text { comparison and follow-up }\end{array}$ \\
\hline Degidi et al. (2003) ${ }^{26}$ & $\begin{array}{l}\text { Study did not define number of missing teeth and there } \\
\text { was no shortened dental arch subgroup for survival results }\end{array}$ \\
\hline Fueki et al. (2015) 27 & Included participants with greater than 10 teeth in study \\
\hline
\end{tabular}


Goshima et al. (2009) ${ }^{28}$

Jepson et al. (2003) $)^{29}$

Mc Kenna et al. (2014) ${ }^{30}$

McKenna et al. (2013) $)^{31}$

Sasse et al. (2014) $)^{32}$

Schmitt et al. (2011) $)^{33}$

Weibrich et al. (2001) $)^{34}$ $\operatorname{arch}(2-12$ missing occlusal units)

Study only presented results with 1 month follow-up

Study did not present a validated oral health related quality

of life outcome

Study presented data on oral health related quality of life

contained in included study

Study only presented results with 1 month follow-up

Mean observation period of study was less than 5 years

Study did not define number of missing teeth or age of

participants

Maximum observation period of study less than 5 years

and there was no shortened dental arch subgroup for

survival results 


\section{Table 3}

Assessment of risk of bias (randomized controlled trials)

\begin{tabular}{|c|c|c|c|c|c|c|c|}
\hline & $\begin{array}{l}\text { Thomaso } \\
\text { net. al } \\
\text { (2007) }\end{array}$ & $\begin{array}{l}\text { Walter } \\
\text { et. al } \\
\text { (2013) }\end{array}$ & $\begin{array}{l}\text { Walter } \\
\text { et. al } \\
\text { (2014) }\end{array}$ & $\begin{array}{l}\text { Wolfart } \\
\text { et. al } \\
\text { (2005) }\end{array}$ & $\begin{array}{l}\text { Wolfart } \\
\text { et. al } \\
\text { (2014) }\end{array}$ & $\begin{array}{l}\text { Wolfart } \\
\text { et. al } \\
\text { (2012) }\end{array}$ & $\begin{array}{l}\text { McKenna } \\
\text { et. al } \\
\text { (2015) }\end{array}$ \\
\hline $\begin{array}{l}\text { Sequence } \\
\text { generation }\end{array}$ & Low risk & Low risk & Low risk & Low risk & Low risk & Low risk & Low risk \\
\hline $\begin{array}{l}\text { Allocation } \\
\text { sequence } \\
\text { concealment }\end{array}$ & Low risk & Low risk & Low risk & Low risk & Low risk & Low risk & Low risk \\
\hline $\begin{array}{l}\text { Blinding of } \\
\text { personnel }\end{array}$ & Low risk & High risk & High risk & High risk & High risk & High risk & High risk \\
\hline $\begin{array}{l}\text { Blinding of } \\
\text { outcome } \\
\text { assessment }\end{array}$ & Low risk & Low risk & High risk & Low risk & Low risk & High risk & Low risk \\
\hline $\begin{array}{l}\text { Incomplete } \\
\text { outcome data }\end{array}$ & Low risk & Low risk & Low risk & Low risk & Low risk & Low risk & Low risk \\
\hline $\begin{array}{l}\text { Selective } \\
\text { reporting }\end{array}$ & $\begin{array}{l}\text { Unclear } \\
\text { risk }\end{array}$ & Low risk & Low risk & Low risk & Low risk & Low risk & Low risk \\
\hline
\end{tabular}

\section{Table 4}

$\underline{\text { Survival of remaining teeth }}$

$$
S D A+R P D \quad S D A \quad C D A
$$

\begin{tabular}{r|lll} 
Walter et & Cumulative & First tooth loss & First tooth loss \\
al. (2013) & Survival & $0.74(0.64,0.84)$ & $0.74(0.63,0.85)$
\end{tabular}




\begin{tabular}{r|llll} 
& $\begin{array}{l}\text { Probability } \\
\text { at 5 years }\end{array}$ & & \\
Gerritsen & Hazard & Anterior teeth & Reference group & Anterior teeth \\
et al. & Ratio (95\% & $1.62(0.29,9.06)$ ) & & \\
(2013) & CI) & Premolar teeth & & $0.22(0.03,1.47) ;$ \\
Gerritsen & Extractions & Upper jaw 0.12 & Upper jaw 0.06 & Upper jaw 0.03 \\
et al. & per year & $(0.12)$; Lower & $(0.08) ;$ Lower jaw & $(0.03)$; Lower \\
(2013) & (Mean (SD)) & jaw $0.06(0.10)$ & $0.05(0.10)$ & jaw $0.03(0.03)$
\end{tabular}

\section{Table 5}

Changes in Oral Health Related Quality of Life

\begin{tabular}{r|llllll}
\multicolumn{5}{c}{} & \multicolumn{5}{c}{ Pre-treatment / Baseline } \\
\hline Wolfart et al. & OHIP-49 & $43.5(18-$ & $31.8(26-$ & $14.7(9-20)$ & $8.3(5-43)$ \\
$(2005)$ & SDA + RPD & SDA & SDA + RPD & SDA \\
& Score & $112)$ & $66)$ & & \\
Wolfart et al. & (Median (IQ & & & & \\
(2014) & range)) & $38.0(14.0-$ & $40.0(18.0$ & $13.0(6.0-$ & $15.5(6.0-$ \\
McKenna et & OHIP-14 & $11.5(4.7)$ & $12.0(5.5)$ & $5.8(3.5)$ & $4.0(2.6)$ \\
al. (2015) & Score (Mean & & & & \\
& (SD)) & & & & &
\end{tabular}




\section{Figure legends}

Figure 1: Percentage of the population aged 60 years or over for the world and regions, $1980-2050^{1}$

Figure 2: Trends in percentage edentate by age: England, 1978-20093

Figure 3: PRISMA flow diagram for studies retrieved through search and selection

Figure 4: Summary of risk of bias (randomized controlled trials)

Figure 5 Meta-analysis of survival of prosthodontic interventions 


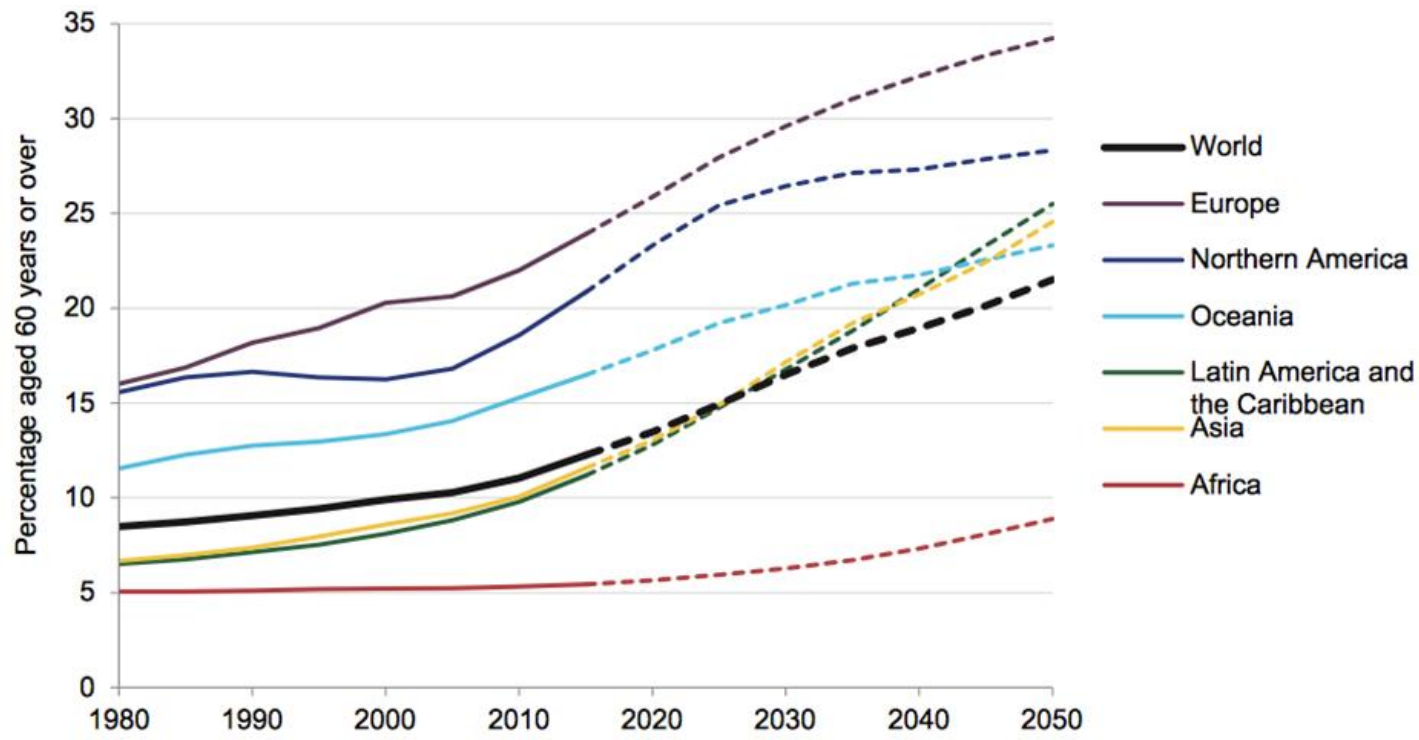




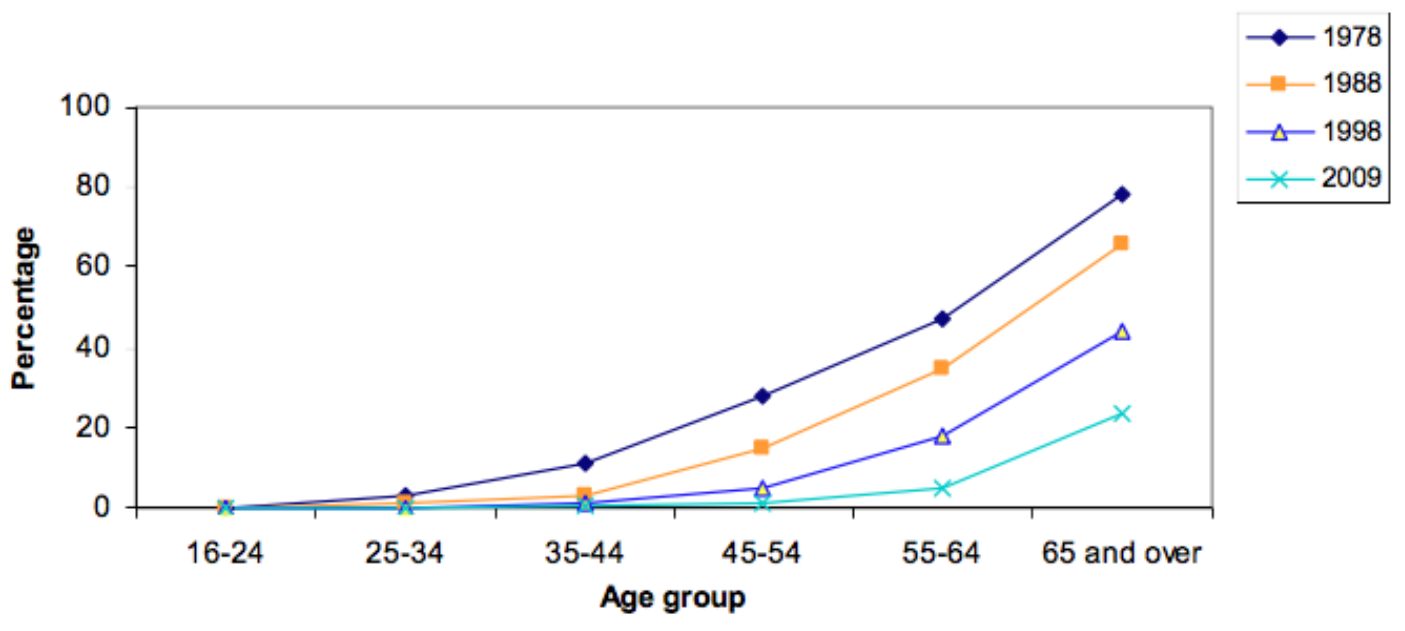




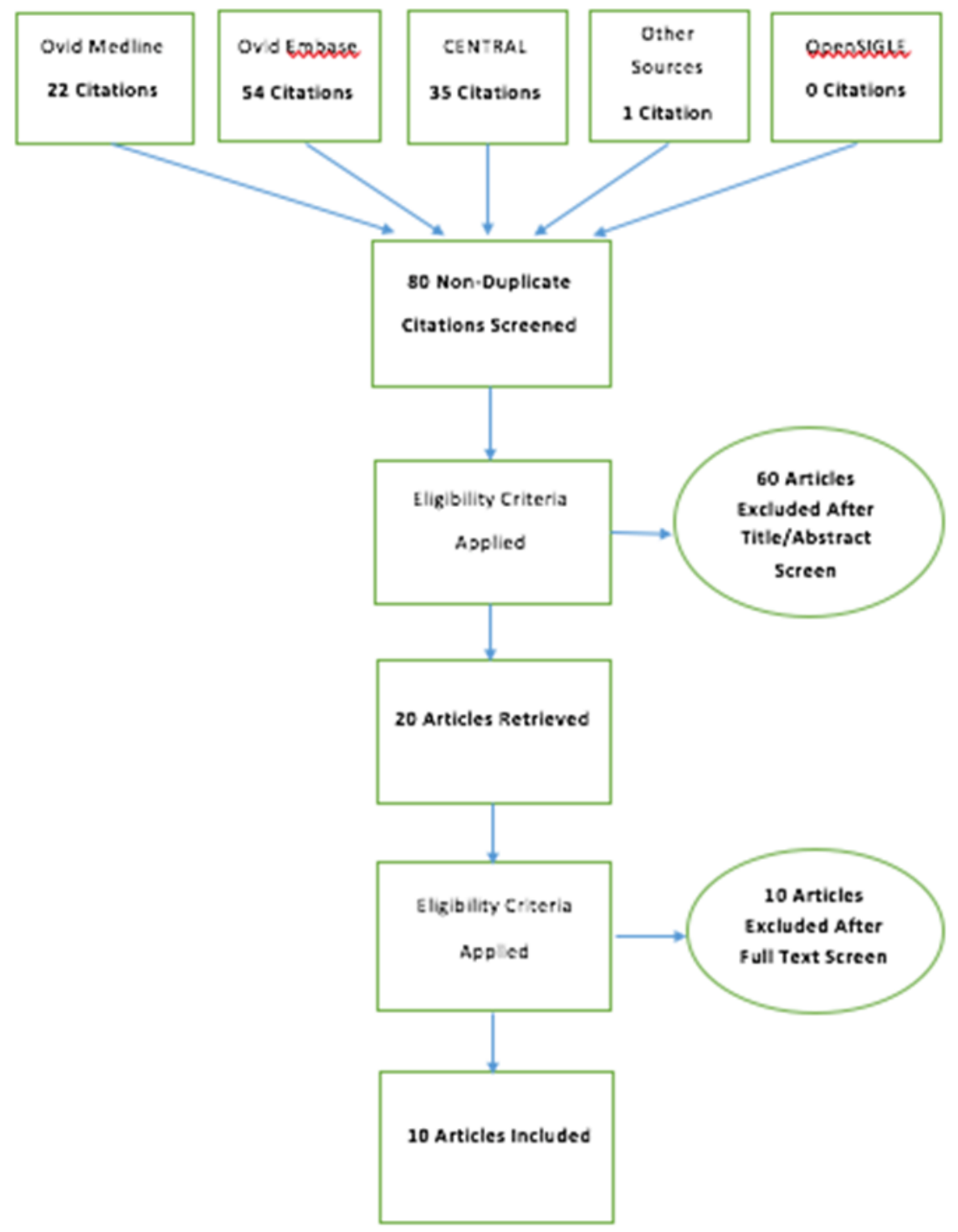




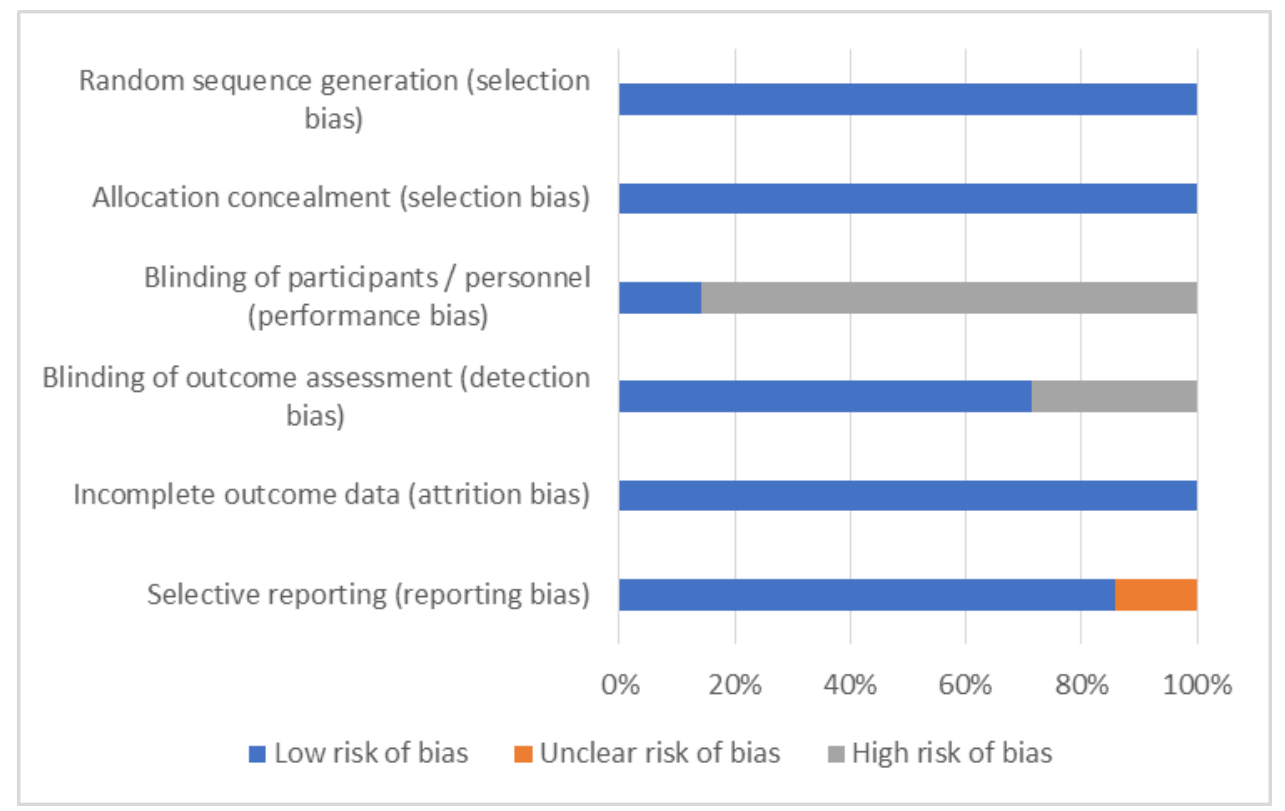


FPDS RPDs Odds Ratio $\quad$ Odds Ratio

Study or Subgroup Events Total Events Total Weight $\mathrm{M}-\mathrm{H}$, Fixed, $95 \% \mathrm{CI}$ $\mathrm{M}-\mathrm{H}$, Fixed, $95 \% \mathrm{CI}$ Budtz-Jorgensen et Thomason et al. (2007)

$8 \quad 41$

Total $(95 \% \mathrm{Cl})$

15

$30 \quad 60.9 \% \quad 0.26[0.10,0.68]$

95

$56 \quad 100.0 \% \quad 0.31[0.15,0.64]$

Total events

$19 \quad 25$

Heterogeneity: $C h i^{2}=0.31, \mathrm{df}=1(\mathrm{P}=0.58) ; \mathrm{I}^{2}=0 \%$

Test for overall effect: $Z=3.17(P=0.002)$

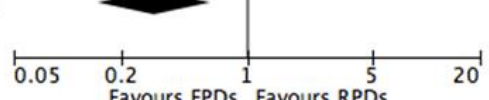

Favours FPDs Favours RPDS 


\section{References}

1. United Nations Department of Economic and Social Affairs Population Division. World Population Ageing 2015. United Nations 2015. New York, NY, USA

2. Watt RG, Steele JG, Treasure ET, White DA, Pitts NB, Murray JJ. Adult Dental Health Survey 2009: implications of findings for clinical practice and oral health policy. British Dental Journal 2013: 214:71-75

3. Steele J G, O’Sullivan I. Executive summary: Adult Dental Health Survey 2009. Health and Social Care Information Centre 2011. London

4. Marcenes W, Kassebaum NJ, Bernabe E, Flaxman A, Naghavi M, Lopez A, Murray CJL. Global Burden of Oral Conditions in 1990-2010: A Systematic Analysis. J Dent Res 2013; 92(7): 592-597

5. Gotfredsen K, Walls AW. What dentition assures oral function? Clin Oral Implants Res 2007; 18 (Supp 3): 34-45

6. Gerritsen AE, Allen PF, Witter DJ, Bronkhorst EM, Creugers NH. Tooth loss and oral health-related quality of life: a systematic review and meta-analysis. Health Qual Life Outcomes 2010; 8: 126

7. Kayser AF. Shortened dental arches and oral function.J OralRehabilitation 1981; 8: 457-462.

8. World Health Organisation. Recent advances in oral health. WHO Technical Report Series No. 826. WHO 1992; 16-17: Geneva

9. Kanno T, Carlsson GE. A review of the shortened dental arch concept focusing on the work by the Kayser / Nijmegen group. J Oral Rehabilitation 2006; 33(11): 850-62

10. Abt E, Carr AB, Worthington HV. Interventions for replacing missing teeth: partially absent dentition. Cochrane Database Syst Rev 2012; 2CD003814.

11. Listl S. Income-related inequalities in Dental Service Utilization by Europeans Aged 50+. J Dent Res 2011; 90(6): 717-723

12. Fueki K, Baba K. Shortened dental arch and prosthetic effect on oral healthrelated quality of life: a systematic review and meta-analysis. Journal of Oral Rehabilitation 2017; 44: 563-572

13. McLister C, Donnelly M, Cardwell C, Moore C, McKenna G. Effectiveness of prosthodontic interventions and survival of remaining teeth in adult patients with shortened dental arches - Protocol for a systematic review and metaanalysis. BDJ Open 2017

14. Moher David, Liberati Alessandro, Tetzlaff Jennifer, Altman Douglas G. Preferred reporting items for systematic reviews and meta-analyses: the PRISMA statement BMJ 2009; 339 :b2535

15. Budtz-Jorgensen E, Isidor F. A 5-year longitudinal study of cantilevered fixed partial dentures compared with removable partial dentures in a geriatric population. J Prosthet Dent 1990;64:42-47

16. Wolfart S., Heydecke G., Luthardt R.G., Marre B., Freesmeyer W.B., Stark H., et al. Effects of prosthetic treatment for shortened dental arches on oral healthrelated quality of life, self-reports of pain and jaw disability: Results from the pilot-phase of a randomized multicentre trial. J Oral Rehabil 2005 November 2005;32(11):815-822.

17. Thomason J.M., Moynihan P.J., Steen N., Jepson NJ. Time to survival for the restoration of the shortened lower dental arch. J Dent Res 2007 Jul 2007;86(7):646-650.

18. Gerritsen A.E., Witter D.J., Bronkhorst E.M., Creugers NHJ. An observational cohort study on shortened dental arches-clinical course during a period of 27-35 years. Clin Oral Investig 2013 2013;17(3):859-866. 
19. Gerritsen A.E., Witter D.J., Bronkhorst E.M., Creugers NH. Increased risk for premolar tooth loss in shortened dental arches. J Dent 201301 Aug 2013;41(8):726-731.

20. Walter M.H., Hannak W., Kern M., Mundt T., Gernet W., Weber A., et al. The randomized shortened dental arch study: Tooth loss over five years. Clin Oral Investig 2013 2013;17(3):877-886.

21. Wolfart S., Muller F., Gerss J., Heyedcke G., Marre B., Boning K., et al. The randomized shortened dental arch study: oral health-related quality of life. Clin Oral Investig 2014 2014;18(2):525-533.

22. Wolfart S, Marre B, Wostmann B, Kern M, Mundt T, Luthardt RG, et al. The randomized shortened dental arch study: 5-year maintenance. J Dent Res 2012 Jul;91(7 Suppl):65S-71S

23. Walter M.H., Marre B., Vach K., Strub J., Mundt T., Stark H., et al. Management of shortened dental arches and periodontal health: 5-year results of a randomised trial. J Oral Rehabil 201401 Jul 2014;41(7):515-522.

24. McKenna G, Allen PF, O'Mahony D, Cronin M, DaMata C, Woods N. The impact of rehabilitation using removable partial dentures and functionally orientated treatment on oral health-related quality of life: a randomised controlled clinical trial. J Dent 2015 Jan;43(1):66-71.

25. Baba K, Igarashi Y, Nishiyama A, John MT, Akagawa Y, Ikebe K, Ishigami T, Kobayashi H, Yamashita S. The Relationship Between Missing Occlusal Units and Oral Health-Related Quality of Life in Patients with Shortened Dental Arches. International Journal of Prosthodontics 2008; 21 (1): 72-74

26. Degidi M, Piatelli A. Immediate Functional and Non-Functional Loading of Dental Implants: A 2- to 60-Month Follow-Up Study of 646 Titanium Implants. Journal of Periodontology 2003; 74: 225-241

27. Fueki K, Igarashi Y, Maeda Y, Baba K, Koyano K, Sasaki K, Akagawa Y, Kuboki T, Kasugai S, Garrett NR. Effect of prosthetic restoration on oral health-related quality of life in patients with shortened dental arches: a multicentre study. Journal of Oral Rehabilitation 2015 42; 701--708

28. Goshima K, Lexner MO, Thomsen CE, Miura H, Gotfredsen K, Bakke M. Functional aspects of treatment with implant-supported single crowns: a quality control study in subjects with tooth agenesis. Clin. Oral Impl. Res. 21, 2010; 108-114.

29. Jepson N, Allen F, Moynihan P, Kelly P, Thomason M. Patient Satisfaction Following Restoration of Shortened Mandibular Dental Arches in a Randomized Controlled Trial. International Journal of Prosthodontics 2003; 16 (4): 409-414

30. McKenna G, Allen F, Woods N, O'Mahony D, Cronin M, DaMata C, Normand C. Cost-effectiveness of tooth replacement strategies for partially dentate elderly: a randomized controlled clinical trial. Community Dent Oral Epidemiol 2014; 42: 366-374

31. McKenna G, Allen F, Woods N, O’Mahony D, DaMata C, Cronin M, Normand C. A preliminary report of the cost-effectiveness of tooth replacement strategies for partially dentate elders. Gerodontology 2013; 30: 207-213

32. Sasse M, Kern M, Marre B, Walter MH. Clinical performance of cantilevered fixed dental prostheses abutments in the shortened dental arch. Journal of Dentistry 2014; 42: 373-376

33. Schmitt J, Wichman M, Eitner S, Hamel J, Holst S. Five-year clinical follow-up of prefabricated precision attachments: A comparison of uni- and bilateral removable dental prostheses. Quintessence Int 2011; 42: 413-418

34. Weibrich G, Buch R, Wegener J, Wagner W. Five-year Prospective Follow-up report of the Astra Tech Standard Dental Implant in Clinical Treatment. The International Journal of Oral and Maxillofacial Implants 2001; 16(4); 557-562 
35. Higgins JPT, Green S (editor). Cochrane Handbook for Systematic Reviews of Interventions Version 5.1.0 (updated March 2011). The Cochrane Collaboration, 2011. Available from www.cochrane-handbook.org

36. Wells G, Shea B, O'Connell D, Peterson J, Welch V, Losos M, Tugwell P: The Newcastle-Ottawa Scale (NOS) for assessing the quality of nonrandomised studies in meta-analyses. 2013. Available from http://www.ohri.ca/programs/clinical epidemiology/oxford.asp,

37. Jepson NJ, Moynihan PJ, Kelly PJ, Watson GW, Thomason JM. Caries incidence following restoration of shortened lower dental arches in a randomized controlled trial. Br Dent J 2001 191:140-144.

38. Jung RE, Zembic A, Pjettursson BE, Zwahlen M, Thoma DS. Systematic review of the survival rate and the incidence of biological, technical and esthetic complications of single crowns on implants reported in longitudinal studies with a mean follow-up of 5 years. Clin Oral Implants Res 2012; 23 (Supp 6): 2-21

39. Pjeturrsson BE, Thoma D, Jung R, Zwahlen $M$, Zembic A. A systematic review of the survival and complication rates of implant-supported fixed dental prostheses (FDPs) after a mean observation period of at least 5 years. Clin Oral Implants Res 2012; 23 (Supp 6): 22-38

40. Pjettursson BE, Tan WC, Tan K, Bragger U, Zwahlen M, Lang NP. A systematic review of the survival and complication rates of resin bonded bridges after an observation period of at least 5 years. Clin Oral Implants Res 2008; 19: 131-141

41. Pjetursson BE, Bragger U, Lang NP, Zwahlen M. Comparison of survival and complication rates of tooth-supported fixed dental prostheses (FDPs) and implant-supported FDPs and single crowns (SCs). Clin Oral Implants Res. 2007 Jun;18 Suppl 3:97-113.

42. King PA, Foster LV, Yates RJ, Newcombe RG, Garrett MJ. Survival characteristics of 771 resin-retained bridges provided at a UK dental teaching hospital. British Dental Journal 2015; 218: 423-428

43. Vermeulen AH, Keltjens HM, Van't Hof MA, Kayser AF. Ten year evaluation of removable partial dentures: Survival rates based on retreatment, not wearing and replacement. Journal of Prosthetic Dentistry 1996 76:3; 267-272

44. Preshaw PM, Walls AW, Jakubovic NS, Moynihan PJ, Jepson NJ, Loewy Z. Association of removable partial denture use with oral and systemic health. Journal of Dentistry 2011:39; 711-719

45. Fuller E, Steele J, Watt R, Nuttall N. Adult Dental Health Survey 2009. The Health and Social Care Information Centre 2011. London, United Kingdom

46. Muller F, Naharro M, Carlsson GE. What are the prevalence and incidence of tooth loss in the adult and elderly population in Europe? Clin Oral Implants Res 18(Supp 3):2-14

47. Holm-Pedersen P, Lang NP, Muller F. What are the longevities of teeth and oral implants? Clin Oral Implants Res 18(Supp 3):15-19

48. Nelson EC, Eftimovska E, Lind C, Hager A, Wasson JH, Lindblad S. Patient reported outcome measures in practice. BMJ 2015;350:g7818

49. Slade GD. Derivation and validation of a short-form oral health impact profile. Community Dentistry and Oral Epidemiology 1997;25:284-90.

50. Gagliardi DI, Slade GD, Sanders AE. Impact of dental care on oral health-related quality of life and treatment goals among elderly adults. Australian Dental Journal 2008;53:26-33.

51. Slade GD, Sanders AE. The paradox of better subjective oral health in older age. Journal of Dental Research 2008;90:1279-85.

52. Faggion CM. The shortened dental arch revisited: from evidence to recommendations by use of the GRADE approach. Journal of Oral Rehabilitation 2011 38; 940-949 
53. Egger, M., Jüni, P., Bartlett, C., Holenstein, F. \& Sterne, J. How important are comprehen- sive literature searches and the assessment of trial quality in systematic reviews? Empirical study. Health Technology Assessment 2003 7: 176.

\section{Appendix 1: OVID MEDLINE / Embase Search Strategy}

1. (t\#\#th* adj6 replac*).mp. [mp=title, abstract, original title, name of substance word, subject heading word, keyword heading word, protocol supplementary concept word, rare disease supplementary concept word, unique identifier]

2. Dental Prosthesis, Implant-Supported/ or Dental Implantation, Endosseous/ or Dental Implants/ or oral implant*.mp.

3. bridge*.mp.

4. Dental Prosthesis, Implant-Supported/ or Denture, Partial/ or Jaw, Edentulous, Partially/ or Denture, Partial, Removable/ or partial denture*.mp. or Denture, Partial, Fixed/

5. 1 or 2 or 3 or 4

6. (short* adj6 dental arch*).mp. [mp=title, abstract, original title, name of substance word, subject heading word, keyword heading word, protocol supplementary concept word, rare disease supplementary concept word, unique identifier]

7. (functional* adj6 dentition*).mp. [mp=title, abstract, original title, name of substance word, subject heading word, keyword heading word, protocol supplementary concept word, rare disease supplementary concept word, unique identifier]

8. 6 or 7

9. $\mathrm{t} \# \#$ th loss.mp

10. surviv*.mp.

11. fail*.mp.

12. "quality of life".mp. or "Quality of Life"/

13. Health Status Indicators/ or Health Status/ or health stat*.mp

14. 9 or 10 or 11 or 12 or 13

15. 5 and 8 and 14

16. limit 15 to (english language and clinical trial, all) 